\title{
Auditoría Medioambiental
}

\author{
Carlos Egúsquiza Pereda \\ Docente de la Facultadi de Ciencias Contables
}

\begin{abstract}
RESUMEN
La presente investigación de Auditoría Medioambiental se halla dentro de la línea de investigación de la AUDITORÍA INTEGRADA O INTEGRAL. Inicialmente se abordaba el tema de la gestión del medio ambiente bajo la denominación de (EVALUACIÓN DE LOS DANNOS DEL MEDIO AMBIENTE», labor realizada hasta ese entonces por biólogos, químicos y ecologistas. Hoy en día se tiene que abordar el tema de gestión del medio ambiente bajo un concepto más amplio e integrador, como es bajo la denominación de Auditoría del Medio Ambiente o Auditoría Ecológica. Vale decir, teniendo en cuenta, la multidisciplinariedad y la transversalidad de los profesionales y técnicos, así como la globalización de la economía, la educación, la ciencia y la tecnología; la problemática de la gestión del medio ambiente conducente a la preservación, protección, control del habitat del hombre en la Tierra, a fin de alargar la corta estadía del hombre en ella, implica la participación de los distintos profesionales y técnicos, principalmente de aquellos que cuenten con la capacidad de gestión, supervisión y control sobre temas del medio ambiente, como ingenieros, biólogos, químicos, economistas, abogados, administradores de empresas y contadores públicos, debido a que estos últimos son profesionales más antiguos en materia de auditoría y por la permanente investigación que vienen efectuando, lo cual les permite involucrarse en un equipo multidisciplinario de profesionales para la ejecución de la AUDITORÍA DEL MEDIO AMBIENTE o AUDITORÍA ECOLÓGICA.
\end{abstract}

En este trabajo se ha abordado la concepción y naturaleza de la auditoría medioambiental que en nuestro medio carece de una concepción clara y precisa, la delimitación de sus características, objetivos, su estructura e importancia. En este artículo se hace resaltar los resultados más sobresalientes y el aporte a la comunidad científica que podrá ser aplicado para efectuar una gestión de calidad y eficiencia de sus actividades o procesos, evitando el daño o la contaminación del medio ambiente por las empresas mineras, industriales, petroleras, pesqueras, madereras, etc.; por ende, será de uso de los profesionales involucrados, firmas consultoras y firmas auditoras.

La auditoría ambiental comenzó en los Estados Unidos de Norteamérica, a fines de los años setenta en el sector industrial, como la industria química, actividad más vulnerable a la contaminación del medio ambiente; sugirieron así los "reglamentos ambientales» para que los gerentes o directivos empezaran a tomarlos en cuenta; extendiéndose a Europa para evaluar a las empresas multinacionales estadounidenses en el cumplimiento de las normas de la preservación del medio ambiente.

Las fuentes preliminares del presente trabajo de investigación están dadas desde el SEGUNDo ConGRESO Federal Guadalajara Jalisco (1992), realizado en la ciudad de México; y el Congreso InternacioSAl De Auditoría InTEgral (1995), realizado en la ciudad de Buenos Aires, en los que se dejaron las bases para que los investigadores y estudiosos en la materia se preocupen de efectuar trabajos en cada -no de los países involucrados. Últimamente, los problemas relacionados con la Auditoría del Medio Ambiente se discutieron en los eventos internacionales de la profesión del Contador Público la XXIII y a XXIV: Conferencia Interamericana de Contabilidad de 1999 y 2001, llevados a cabo en San juan de Puerto Rico y Punta del Este, Uruguay, respectivamente; en los que se esbozaton la necesidad de continuar con el estudio en el campo de medio ambiente, a la par con la globalización económica, Jentífica y técnica. 
En el Perú, cası no se ha avanzado con respecto a los temas de gestión del medio ambiente, ni mucho menos con respecto a las acciones de supervisión y control de las empresas o instituciones que dañan el habıtat del hombre, más aún, no se tiene institucionalızada ni mucho menos organizada una actividad de alta especialización, como es la auditoría del medio ambiente, como tema de la auditoría integral o integrada, con enfoque multidisciplinario, ni instituciones especializadas, como el caso de Méxıco, que cuenta con un organismo denominado «Procuraduría Federal de Protección al AmbientePROFEPA", que se encarga de asignar un instrumento para proteger o mejorar el entorno ambiental e incentivar a las empresas a la utilización de un instrumento valioso de la gestión del medio ambiente. Asımısmo, en BolrviA se viene trabajando el proyecto de «Protección Ambiental en la Industria Boliviana -PAIB»; en Colombia existen investigaciones realizadas en la Universidad Cooperativa de Colombia (UCC) -seccional Bucaramanga- sobre contabilidad ambiental, como una opción para dar respuesta a la necesıdad de contar con información y parámetros que hagan posible evaluar la sostenibilidad ambiental dentro del proceso de desarrollo de las naciones, y así cumplir con lo pactado en la Cumbre de Río de Janeıro en el año 1992. En la Argentina se lleva a cabo a través de la Secretaría de Ambiente y Desarrollo Sustentable, que ha desarrollado un Sistema de Indicadores de Desarrollo Sostenible y el Programa ENGIRSU (Estrategia Nacional para la Gestión Integral de Residuos Sólidos Urbanos).

Palabras clave: Auditoría del Medio Ambiente, Auditoría Ecológica, Gestión del Medio Ambiente, Desarrollo Sostenible, Conservación del Medio Ambiente.

\section{INTRODUCCIÓN}

La gestión de la conservación del medio ambiente no solo implica el control y supervisión de los procesos o actividades que dañan el habitad del hombre, sino implica que los Estados promuevan o fomenten el desarrollo de una cultura de preservación del medio ambiente. En el Perú no existen instituciones especializadas, como el caso de México; por ejemplo, no se utiliza un instrumento valioso de la gestión del medio ambiente, como es la Auditoría del Medio Ambiente o auditoría medio AMBIENTAL, o simplemente AUDITORÍA ECOLÓGICA.

En el Perú casi no se ha avanzado con respecto a los temas de gestión del medio ambiente, ni mucho menos con respecto a las acciones de supervisión y control de la empresas o instituciones que dañan el hábitat del hombre -planeta Tierra-; aún más, no se tiene institucionalizado, ni mucho menos organizado, una actividad de alta especialización, como es la auditoría del medio ambiente, como tema de la auditoría integral o integrada, con enfoque multidisciplinario e interdisciplinario.

En esta oportunidad, nos hemos dedicado a la investigación sobre auditoría del medio ambiente; por ello, el rol de los contadores públicos es afrontar los nuevos retos del siglo XXI, las nuevas exigencias de la globalización en la economía, la educación y la gestión de los conocimientos; por tratarse, aún más, que el presente tema, en el Perú es de reciente divulgación y de interés para los distintos profesionales, estudiosos e investigadores, a que el paso tan raudo por nuestro planeta nos obliga a contribuir a que esta estadía tan corta sea más duradera; por ello, es de preocupación de todos nosotros la preservación y conservación del medio ambiente.

La auditoría del medioambiente en nuestro medio no es de aplicación institucionalizada, ya que no existen instituciones o sociedades especializadas encargadas de hacer cumplir las distintas disposiciones 
.egales sobre preservación del medio ambiente, como en otros países, compuesta oor entidades privadas y el Estado. En estos estados, no se acepta ningún producto sin la certificación respectiva, dada como c onsecuencia del proceso de auditoría del medio ambiente o ecológica; por ejemplo: LSA, México, etc.

La Auditoría del Medio Ambiente se ha convertido en sinónimo de responsabilidad rganizacional con la agenda verde y es un area creciente, tanto en complejidad como en importancia ${ }^{1}$.

Indudablemente que una de las - erramientas valiosas para el proceso de la auditoría del medio ambiente es el de aplicación de la norma ISO 14000.

Para el proceso de esta investigación se ha recurrido a instituciones y empresas que se hallan vinculadas con la protección del medio ambiente:

1. En el Perú: Instituto de Recursos Naturales - INRENA, Consejo Nacional del Medio Ambiente-CONAM, Universidad Nacional Agraria La MolinaUNALM, Contraloría General de la República, Sociedad Nacional de Industrias, colegios profesionales relacionados, Instituto de Investigaciones de la Selva, etc.

2. En la ciudad de México: Procuraduría Federal de Protección del Medio Ambiente-PROFEPA; que se encarga de crear un novedoso instrumento para mejorar el entorno ambiental e incentivar a las empresas a la utilización de AUditoría AMBIENTAL.

3. Unión Europea-UE, Eco. Management and Audit. Scheme (EM-AS).
4. British Standards Institute (BSI)-BS (UE).

5. En Bolivia: Proyecto de Protección Ambiental en la Industria Boliviana«PAIB».

6. Ecuador: Ministerio de Energía y Minas, Auditoría Ambiental: «Construcción del Oleoducto de Crudos Pesados».

7. Foro Boliviano sobre Medio Ambiente y Desarrollo-FOBOMADE, etc.

Las exigencias del mundo globalizado y competitivo del siglo XXI como los avances económicos, culturales, científicos y tecnológicos nos obligan a realizar estas investigación en materia de auditoría en temas novísimos como es la AUDITORÍA DEL MEDIO AMBIENTE. Estas exigencias hacen que los profesionales dedicados a la actividad de auditoría estemos obligados a continuar investigando y explorando la aplicación de la auditoría a nuevos campos o temas, no precisamente a los que tienen intima relación con el campo del contador público o aquellos temas relacionados con los enfoques contables o de auditoría contable o financiera. Por ello, implicará la adopción de filosofías y políticas tendentes a la cultura de la preservación del medio ambiente y, su respectiva supervisión y control de las actividades del individuo, empresas o instituciones sobre sus actividades, lo cual pueden hacer que estemos acortando nuestra corta estadía por este planeta.

En el presente estudio realizaremos una descripción del tema, se detallarán los antecedentes encontrados sobre Auditoría del Medio Ambiente, y un completo marco teórico que abarca reseña histórica, conceptos, naturaleza, objetivos, impor-

GRAY, Rob y otros. Contabilidad y Auditoria Ambiental, p. 83. 
tancia, características, ventajes, estructura, normas y organismos responsables en el Perú de la Auditoría del Medio ambiente. Por último, se presentarán los resultados más saltantes, el impacto y aporte de la presente investigación, recomendaciones y conclusiones.

\section{PLANTEAMIENTO DELESTUDIO}

En el Perú y Latinoamérica, en especial en países subdesarrollados, no se tiene debidamente establecida la GESTIÓN Y AUDITORÍA DEL MEDIO AMBIENTE, las cuales Se hallan en proceso de estudio e implementación, no existiendo trabajos o investigaciones hechas que orienten a la concepción racional y científica. Por ejemplo, los temas:

a. Filosofía o cultura de la gestión medio ambiental.

b. Balance financiero-económico de los daños del medio ambiente.

c. Objetivos precisos con respecto a la protección o preservación del medio ambiente.

d. Solución de problemas ecológicos.

e. Bosquejo y planteamiento de la auditoría para la protección del hábitat del hombre.

f. Naturaleza, contenido y proceso de la auditoría.

g. Técnicas y procedimientos aplicables.

h. Metodología a ser utilizada en la redacción de los informes de auditoría ecológica.

1. Búsqueda y evaluación de las evidencias de auditoría, etc.

Los resultados de la presente investigación serán aplicables por la empresas e instituciones, que en sus actividades o procesos dañan o contaminan el medio ambiente, tales como: empresas mineras industriales, petroleras, pesqueras. madereras, químicas, etc. También esta relacionado con los problemas de «contaminación, a estos se incluye el problema de la basura, contaminación del aire, los ruidos, la contaminación del agua, los desechos de las fábricas, el problema del control del higiene y otross; es decir, todo lo relacionado a la flora y fauna, así como con la capa de ozono. En tal sentido, será de aplicación por los profesionales y firmas consultoras dedicados a la gestión y auditoría ecológica, como: ingenieros, biólogos, químicos, contadores públicos, economistas, abogados, administradores de empresas, etc.

\section{DESCRIPCIÓN DEL ESTUDIO}

\section{a. Antecedentes}

En el Perú, a pesar de que contamos con cifras alarmantes de contaminación del medio ambiente, ya que según el último reporte de la Unión Mundial de Conservación (UICN) del 2006: en el Perú, de un total de 3115 especies incluidas en la lista roja del 2006, se ha registrado la extinción de 2 especies (una en flora y otra en fauna), se han reportado 45 especies en peligro crítico ( 36 de fauna y 9 de flora), y 90 en peligro ( 75 de fauna y 15 de flora) ${ }^{2}$. Cası no se ha avanzado con respecto al tema de gestión del medio ambiente, mucho menos en lo que se refiere al control y la supervisión del mismo; solo la Contraloria General de la República lo está efectuando muy superficialmente cuando se trata de empresas estatales; recién con la

2 ECO FLO ES, Ca O «Ecosistemas peruanos se encuentran amenazados por el avance del hombre». En: El Comercio. 20-05-2006. p B 2 
emisión de la Ley N. ${ }^{\circ}$ 27066, del 13 de febrero de 1999, se ha visto formalizada sus actividades en materia de auditoría ambiental, que le otorga la atribución legal para efectuar auditorías ambientales e informar sobre sus resultados periódicamente al Congreso de la República. Su instalación como unidad orgánica se produce a partir de la emisión del Reglamento de Organización y Funciones, aprobado por Resolución de Contraloría 071-2000-CG, del 3 de abril de 2000.

En el caso de la Contraloría, la auditoría no está dirigida a los verdaderos entes que causan el daño al medio ambiente, como son las empresas, solo lo realizan de manera general y abstracta, a diferencia de México que realiza el control y supervisión directamente a las empresas y, además, evalúan los daños y los hace responsables de los mismos.

La Contraloría General, desde el año de 1998, ya había constituido un grupo de trabajo que realizó la auditoría de gestión ambiental (piloto) en el Bosque Nacional Alexander Von Humboldt (Pucallpa), en la amazonía peruana. Esta acción de control contó con la colaboración de funcionarios del Tribunal de Cuentas de la Unión del Brasil. En el año de 1999 se efectuaron 17 auditorías de gestión ambiental en la cuenca del río Mantaro -ubicada entre los departamentos de Pasco y Junín, en la cordillera andina peruana-, la cual contó con la participación de auditores de Colombia, Honduras, México, Brasil, Nicaragua, Chile y Panamá. Esta experiencia permitió consolidar la metodología que venía siendo utilizada, la misma que posteriormente se traduce en la Guía Metodológica de Auditoría de Gestión AMBIENTAL, aprobada mediante Resolución de Contraloría N. ${ }^{\circ} 112-2000-C G$, del 22 de junio del 2000. Mediante Resolución de Contraloría N. ${ }^{\circ}$ 345-2002-CG del 30 de diciembre de 2002, se crea la Gerencia de Medio Ambiente y Patrimonio CulturalMAC, que tiene como finalidad planificar, organizar, dirigir, ejecutar y evaluar las acciones de control y actividades de instituciones públicas descentralizadas, órganos desconcentrados, órganos de línea y proyectos de inversión a nivel nacional, que se encuentran bajo suámbito de control; así como efectuar auditorías de medio ambiente y de patrimonio cultural, priorizando zonas críticas (entre otros, cuencas hidrográficas, áreas naturales protegidas, sitios arqueológicos y convenciones ambientales internacionales), donde se busca abordar la problemática de manera integral. $^{3}$

Según Rob Gray, ${ }^{4}$ la Auditoría del Medio Ambiente se ha convertido en sinónimo de responsabilidad organizacional con la agenda verde y es un área creciente, tanto en complejidad como en importancia.

Indudablemente, una de las herramientas valiosas para el proceso de la auditoría del medio ambiente es la aplicación de la norma ISO 14000, la cual, no es una sola norma, sino que forma parte de una familia de normas que se refieren a la gestión ambiental aplicada a la empresa, cuyo objetivo consiste en la estandarización de formas de producir y prestar servicios que

Contraloría General de República. «Medio'Ambiente y Patrimonio Cultural». [En línea]. Disponible en: http://www.contraloria.gob.pe/ma/index.htm

- GRAY, Rob; BEBBINGTON, Jan y WALTERS, Diana. Contabilidad y Auditoria Ambiental. Bogotá, ECOE Ediciones, 1999. 
protejan al medio ambiente, aumentando la calidad del producto y como consecuencia la competitividad del mismo ante la demanda de productos cuyos componentes y procesos de elaboración sean realizados en un contexto donde se respete al ambiente; además del su conocimiento, por parte de las organizaciones, es más importante la implementación y actualización de este tipo de reglamentación; pues con base en ella, las organizaciones pueden optimizar y mejorar todos sus procesos productivos y reducir el impacto negativo que causan en el medio ambiente del cual se proveen. Estas normas forman parte además de la serie ISO (International Standart Organization) de donde provienen las conocidas ISO $9000 \mathrm{e}$ ISO 9001 , referidas estas ultimas a la calidad total dentro de la empresa ${ }^{5}$.

Para el proceso de esta investigación, se ha recurrido a instituciones y empresas que se hallan vinculadas con la protección del medio ambiente:

1. En el Perú: Instituto Nacional de Recursos Naturales -INRENA, Consejo Nacional del Medio Ambiente-CONAM, Universidad Nacional Agraria La MolinaUNALM, Contraloría General de la República, Sociedad Nacional de Industrias, colegios profesionales relacionados, Instituto de Investigaciones de la Selva, etc.

La Intendencia de Áreas Naturales Protegidas de INRENA es el órgano encargado de la adecuada gestión de las áreas naturales protegidas que conforman el Sistema Nacional de Áreas Naturales Protegidas por el Estado SINANPE y la supervisión de las áreas que no forman parte de este sistema: áreas de conservación regional, municipal y privadas, incluyendo sus zonas de amortiguamiento, con el objetivo de proteger la diversidad biológica, a través de la provisión de bienes y servicios que contribuyen al desarrollo sostenible del país como un legado para las futuras generaciones.

En el Perú, la superficie protegida actualmente por el Sistema Nacional de Áreas Protegidas -SINANPE es de 17660 211.88 Has., con un total de 61 áreas naturales protegidas que abarcan cerca del $13.74 \%$ de la superficie total del país, lo cual coloca al Perú entre los países de América Latina con mayor porcentaje de territorio conservado bajo esta modalidad. Las áreas naturales protegidas son lugares especialmente valiosos para la conservación de la naturaleza, por lo que se encuentran sujetas a un régimen de protección legal por el Estado, a fin de garantizar el logro de sus objetivos.

El CONAM se encuentra realizando programas nacionales de supervisión y control en diferentes aspectos, tales como: biodiversidad, biocomercio, bioseguridad, calidad del aire, calidad ambiental y ruido. Asimismo, se encuentra realizando el Programa Nacional de Residuos Sólidos y el Programa de Ciudadanía Ambiental ${ }^{6}$.

5 CASCIO,Joseph; WOODSIDE, Gayle y MITCHELL, Philip. Guia ISO 14000: las nuevas normas internacionalespara la administración ambiental. México, McGraw-Hill, 1997,pp. 3-4

6 EI Consejo Nacional del Medio Ambiente - CONAM [En línea]. Disponible en: http://www.conam.gob.pe/ modulos/home/queeselconam.asp 
2. British Standards Institute (BSI) - BS (UE).

3. En México existe la Procuraduría Federal de Protección del Medio AMBIENTE - PROFEPA, institución que se encarga de crear un instrumento para mejorar el entorno ambiental e incentivar a las empresas a la utilización de Auditoría Ambiental, la cual otorga la Certificación de Industria Limpia, que tiene por objetivos:

- Proteger el ambiente y fomentar el desarrollo de una cultura ambiental empresarial.

- Motivar en los consumidores el hábito de adquirir productos fabricados por Industrias que observen prácticas de cuidado ambiental.

- Distinguir a las empresas que a través de la auditoría, y con el respectivo plan de acción, han aceptado la responsabilidad voluntaria de proteger a sus trabajadores, a la comunidad y al ambiente mediante el otorgamiento del certificado como Industria Limpia.

- La vigencia del certificado será de un año.

Los resultados han sido:

- A la fecha se han concertado 1150 auditorías de las empresas (de éstas, sólo se han concluido $1077 ; 73$ se encuentran en proceso; y de las concluidas, sólo se han firmado 504 convenios de concertación para invertir 6500 millones de pesos [16 millones / empresa]).

- Las principales empresas que han terminado el Plan de Acción concertado, haciéndose acreedoras al Reconocimiento mediante el Certi- ficado de Industria Limpia son: CFE, PEMEX, FNM, en el sector público; y en el privado: Cemex, GM, PEÑOLES, FORD.

4. En Bolivia se ha realizado el Proyecto de Protección Ambiental en la Industria Boliviana - PAIB; existe, además, un Consejo Boliviano para la Certificación Forestal Voluntaria - CFV y el Foro Boliviano sobre Medio Ambiente y Desarrollo - FOBOMADE, etc.

El Consejo Boliviano para la Certificación Forestal Voluntaria-CFV es una asociación civil nacional sin fines de lucro, creada formalmente en Santa Cruz, Bolivia, en junio de 1995. Su misión es proveer la aplicación práctica del manejo forestal mediante la promoción eimplementación del proceso de certificación. El CFV no certifica productos forestales. Es responsable de mantener y de garantizar la credibilidad del sistema a nivel nacional.

5. En Ecuador, una de las instituciones encargadas para tal fin es el Ministerio de Energía y Minas, el cual ha realizado el proyecto de "Construcción del Oleoducto de Crudos Pesados». Asimismo, el Ministerio del Ambiente de la República del Ecuador, el cual tiene los siguientes proyectos en ejecución:

- BID ATN/SF 8170-EC. Programa de desarrollo sostenible de la Frontera Sur.

- BID ATN/SF-8182-EC. Desarrollo del Sistema Descentralizado de Gestión Ambiental.

- BID-GALAPAGOS. Programa de Manejo Ambiental de las Islas Galápagos. 
- CAMAREN. Capacita a técnicos y promotores en el manejo de los recursos naturales.

- CiUdAdANía AmBIENTAL. Programa que fortalece la participación en la gestión ambiental.

- ECU/00/G31. Control de Especies Invasoras en el Archipiélago de Galápagos.

- GEF-COPs. Desarrollo del Plan Nacional de Implementación para la Gestión de los Contaminantes Orgánicos Persistentes en Ecuador.

- MAE-HOLANDA. Fortalecimiento del MAE para el ejercicio de sus competencias como autoridad ambiental.

- Marco Nacional de Seguridad de la Biotecnología.

- NCSA. Autoevaluación Nacional de Capacidades.

- VIGILANCIA VERDE. Cuerpo de Control Forestal y Vida Silvestre integrado por organizaciones públicas y privadas.

Y los siguientes proyectos ejecutados:

- BIDATN/SF-6798-EC. Programa de Fortalecimiento Institucional para la Administración Ambiental.

- GEF-PNUD ECU/99/G31. Preparación de la Comunicación Nacional ante la Convención Marco de Naciones Unidas sobre Cambio Climático.

- PETRAMAZ. Contribuye a la conservación y manejo racional de áreas protegidas.

UCP-PATRA. Proyecto integral de gestión ambiental en el ecuador.

UNITAR. Desarrollo y Mantenimiento de un Programa Nacional para la Gestión Racional de las Sustancias Químicas.

- UNitAR RETC. Registro de Emisiones y Transferencias de Contaminantes en el Ecuador ${ }^{\top}$.

6. En la Argentina tenemos la Secretatía de Ambiente y Desarrollo Sustentable, quienes han desarrollado un Sistema de Indicadores de Desarrollo Sostenible (agosto de 2005) y el Programa ENGIRSU (Estrategia Nacional para la Gestión Integral de Residuos Sólidos Urbanos).

7. En Cuba existen varios organismos que se encargan del medio ambiente, como son:

- Agencia del Medio Ambiente- AMA, la cual dirige, ejecuta, coordina y controla acciones, proyectos y programas especializados que garanticen la gestión ambiental para lograr el cumplimiento de la Ley de Medio Ambiente, la Estrategia Ambiental Nacional y el Programa Nacional de Medio Ambiente y Desarrollo. Realıza la inspección estatal ambiental; controla y exige por la protección del medio ambiente y el uso racional de los recursos naturales. Integra y ejecuta planes y programas de investigación, investigacióndesarrollo y servicios científicotécnicos vinculados a la gestión ambiental, al estudio sistemático de los recursos naturales y de otras esferas de interés.

CITMA. Ministerio Ciencia y Tecnología del Medio Ambiente; el cual es el organismo encargado de dirigir, ejecutar y controlar la política

Ministerio del Ambiente de la República del Ecuador. [En línea]. Disponible en: http://www.ambiente.gov.ec/ 
del Estado y del Gobierno en la actividad científica y tecnológica, la política ambiental y de uso pacífico de la energía nuclear, propiciando su integración coherente para contribuir al desarrollo sostenible del país.

- CIGEA. Centro de Información, Gestión y Educación Ambiental. Institución que dirige, controla y promueve la gestión ambiental orientada al uso racional de los recursos naturales, la protección y conservación de los ecosistemas y la disminución de la contaminación, en función de proteger el medio ambiente, contribuyendo al desarrollo económico y social de forma sostenible. Garantiza la educación ambiental, la divulgación, el manejo de datos e informaciones sobre el medio ambiente y la información ambiental especializada, en el marco de la Estrategia Ambiental Nacional y la Estrategia Nacional de Educación Ambiental ${ }^{8}$.

8. En Brasil se tiene el Fondo Nacional de Medio Ambiente de Brasil (FNMA), el cual es una institución pública bajo el control del Ministerio del Medio Ambiente, que fue creada en 1989 como una de las primeras acciones del Gobierno brasileño para financiar proyectos ambientales que promuevan el uso racional de los recursos naturales y el mantenimiento, mejoría o recuperación de la calidad ambiental de los diferentes ecosistemas brasileros. La capitalización del Fondo se ha realizado, a diferencia de muchos fondos ambientales, a través de la firma de dos Acuerdos de Préstamos con el Banco Interamericano de

\footnotetext{
http://wwwmedioambiente.cu/
}

Desarrollo, BID, y la contrapartida del Tesoro Nacional de Brasil. Uno de los retos institucionales que el FNMA ha identificado para su gestión en el corto y mediano plazo es la descentralización de sus funciones a través de socios regionales y el establecimiento de mecanismos de evaluación de impacto que le permitan medir la contribución del Fondo al uso racional de los recursos naturales en Brasil.

9. En Colombia, la Universidad Cooperativa de Colombia (UCC) -seccional Bucaramanga-, sobre contabilidad ambiental trabaja como una opción que ofrece respuesta a la necesidad de contar con información y parámetros que hagan posible evaluar la sostenibilidad ambiental dentro del proceso de desarrollo de las naciones, y así cumplir con lo pactado en la cumbre de Río de Janeiro en el año 1992. Evento en el que no solo se asume la responsabilidad política del desarrollo sostenible, sino que se recomienda, entre otros, la incorporación de la "contabilidad ambiental" al sistema de cuentas nacionales. Sin embargo, a la fecha se ha avanzado poco en esa materia, adoleciendo de herramientas $e$ instrumentos para medir y evaluar de manera integral los avances en la sostenibilidad en los procesos de desarrollo de las naciones.

10.Unión Europea-UE, Eco. Management and audit. Scheme (EM-AS). En Europa su introducción aún puede considerarse reciente puesto que tiene sus origenes en los años 80 , con diferentes énfasis según los países. Merece subrayar el hecho de 
que en los países al Norte de Europa es donde existe una mayor sensibilización; por ejemplo, fue Holanda el primer país europeo que incorporó las auditorías medioambientales como herramientas de gestión medioambiental, pretendiendo, además, fijar la obligatoriedad de la realización de auditorías externas para determinadas empresas.

El desarrollo de la auditoría medioambiental en España es semejante, en cuanto a motivación, a los desarrollados en Estados Unidos y al resto de la Unión Europea, aunque también ha sido utilizada para otros fines como pueden ser: la agilización de trámites adminıstrativos y concesión de ayudas. subvenciones e incentivos para llevar a cabo mejoras en la empresa; justificar $r$ divulgar a organismos interesados la metodología de la gestión en la empresa; divulgar, cuando interesa, la situación medio ambiental de la empresa y fijar primas de seguros que cubran los riesgos del deterioro ambiental.

11.En EE.UU. y Europa los delitos ecológicos son castigados con multas muy elevadas, se tienen los siguientes casos:

\begin{tabular}{|c|c|c|}
\hline EMPRESA & LUGAR & DELITO \\
\hline $\begin{array}{l}\text { Empresa Imperial Che- } \\
\text { mical Industries - ICI }\end{array}$ & EE.UU. & $\begin{array}{l}\text { Causar contaminación por una } \\
\text { de sus instalaciones de } \\
\text { Lousiana. }\end{array}$ \\
\hline Empresa Monsanto & EE.UU. & $\begin{array}{l}\text { Ocasionar fuga de ácido en su } \\
\text { factoría de Massachussets. }\end{array}$ \\
\hline Empresa Shell & Europa & $\begin{array}{l}\text { Vertir petróleo al Río Mersey } \\
\text { del Reino Unido, con graves } \\
\text { consecuencias para la flora y } \\
\text { fauna de la zona. }\end{array}$ \\
\hline EmpresaSandoz & Europa & $\begin{array}{l}\text { Contaminar el rio Rhin con } \\
\text { pesticidas. }\end{array}$ \\
\hline Empresa papelera Tipel & España & $\begin{array}{l}\text { Realizar en repetidas ocasiones } \\
\text { vertidos ilegales al Río } \\
\text { Congost. }\end{array}$ \\
\hline
\end{tabular}

En nuestro medio no existe un estudio adecuado y científico, ya que revisando la información bibliográfica solo hemos encontrado artículos publicados en distintas revistas de las universidades del país; por lo que hemos efectuando la presente investıgación, desde la perspectiva científicotécnica, para cubrir las necesidades de gestión, supervisión de las instituciones, empresas y personas involucradas, a fin de contribuir a la prevención de la contaminación del medio ambiente, para que la corta estadía del hombre en la tierra sea más prolongada.

\section{b. Hipótesis}

En el estudio de investigación indicada se ha planteado las siguientes hipótesis:

H1. La auditoría del medio ambiente en nuestro medio carece de una concepción clara y precisa con respecto a naturaleza, contenido, amplitud y profundidad de las pruebas. 
H2. Los entes involucrados (personas y empresas) no están debidamente informados de la importancia $y$ trascendencia de la auditoría del medio ambiente.

H3. Existe la carencia de una formación académica en cuanto al tema materia de estudio, mientras en las currículas de estudios de la universidades del país no se incluyen asignaturas con respecto a la preservación y auditoría ecológica o del medio ambiente.

H4. Las personas y empresas involucradas no realizan auditorías del medio ambiente por falta de participación del Estado, como ente de control y supervisor mediante la obligatoriedad de llevar a cabo la auditoría del medio ambiente.

\section{MARCO TEÓRICO E INSTRU- MENTOS CIENTÍFICO Y TÉCNICO ACERCA DE LA AUDITORÍA DEL. MEDIO AMBIENTE}

\section{Reseña histórica de la Auditoría Medioambiental}

La expresión «Auditoría Social»-proveniente de un concepto cronológicamente anterior, la Contabilidad Social y ésta, a su vez, ligada al de Responsabilidad Social de la Empresa- fue introducida en la literatura profesional por primera vez en 1953, por el Prof. Howard R. Bowen, en su obra Social Responsibilities of the Businessman.

Para fines de la década de los setenta, ya se reconocía en los Estados Unidos, pionero mundial por entonces en esta materia, al menos cinco actividades de carácter social en la vida empresarial:
- Responsabilidad frente a los recursos humanos de la empresa;

- Consideración y atención a las minorias existentes en la región o país donde operaba la empresa;

- Calidad y seguridad de los productos a la venta;

- Participación en las actividades de la comunidad o comunidades en que la empresa desarrollaba sus actividades;

- Protección del medio ambiente.

La preocupación de las empresas por la problemática del medio ambiente se centralizaba en:

- Actividades dirigidas a aliviar o prevenir el deterioro del medio ambiente;

- Equipos necesarios para combatir la contaminación;

- Costos de los programas para luchar contra la polución;

- Esfuerzos para reducir los efectos contaminantes de sus productos;

- Esfuerzos para fomentar la idea del reciclaje de los materiales de desecho.

En realidad, las actividades orientadas a preservar el medio ambiente siempre se han dado; sin embargo, los primeros y funda-mentales actos destinados a la conservación del medio ambiente surgen en 1972, año en que se realizó, en Estocolmo, la Conferencia Mundial sobre el Medio Humano de las Naciones Unidas. A partir de este evento, los temas medio ambientales calan en la conciencia del mundo y adquieren una importancia notable.

Posteriormente, y luego de transcurrido veinte años (en junio de 1992), las Naciones Unidas celebró en Río de Janeiro (Brasil), la Conferencia sobre Medio Ambiente y Desarrollo, evento al que se le denominó «La 
cumbre de la Tierran, y en la que se aprobó una declaración sobre los derechos y responsabilidades de los países con relación al medio ambiente. Esta declaración contiene dos aspectos fundamentales: el permanente deterioro del medio ambiente y su capacidad para sostener a la vida, y la necesidad de implantar políticas, a fin de que el progreso económico deba realizarse coordinadamente con acciones de protección del medio ambiente

\section{Concepto de Auditoría Ambiental}

Para el desarrollo de este punto podemos citar a los trabajos realizados por la Procuraduría Federal de Protección del Medio Ambiente- PROFEPA del gobierno Mexicano y otros autores contrastados.

Según PROFEPA la rA Auditoria Ambiental es una evaluación objetiva de los elementos de un sistema para determinar si son adecuados y efectivos para proteger al medio ambiente. El sistema incluye las actividades que por su naturaleza constituyen un riesgo potencial para el medio ambiente y las derivadas de las medidas para prevenir o actuar en caso de contingencias o emergencias ambientales, el personal involucrado y los conceptos que los norman y se contienen en documentos aceptados por la empresay.

Según Uñi Ugaz, la auditoría del medio ambiente (ADELMA) como el proceso de investigación por un auditor independiente dirigido a determinar el grado de eficiencia empresarial, con relación al grado de satisfacción experimentado por la comunidad y su babitat, señalado en su informe de auditoría a los agentes degradantes del medio ambiente y la magnitud de la degradación producidas?.
Armando Miguel et al. define la Auditoría Ambiental como: «Una Investigación sistemática, ejecutada por especialistas, destinada al sistema de gestion ambiental y sus resultados. Es un instrumento de la administración de la empresa que se ocupa de las rutinas de trabajo y procedimientos de ésta o de uno de sus sectores, referidos a la gestión ambiental, al nivel de cumplimiento con las leyes ambientales y la política de la empresa misma ${ }^{10}$.

Telma Malheiros la conceptualiza como "Un procedimiento ordenado que tiene por objetivos básicos el examen y evaluación, periódica $u$ ocasional de los aspectos legales, técnicos y administrativos relacionados a las actividades ambientales de una empresa, como un instrumento de análisis de su desempeño ambiental y de las acciones relativas a esos aspectos»".

Asimismo, para Valle, la Auditoría Medioambiental «Es un instrumento de gestión que permite bacer una evaluación sistemática, periódica, documentada y objetiva de los sistemas de gestión $y$ de desempeño de los equipos (equipamientos) instalados en el establecimiento de una empresa, por fiscalizar y limitar el impacto de sus actividades sobre el medio ambiente» ${ }^{12}$.

Para Gonzáles Malaxechevarria «Es aquel componente o compartimiento de la Auditoria Social que consta en un examen y evaluación independiente, sistemático, periódico, documentado y objetivo, realizado por un equipo interdisciplinario de auditores ambientalistas (profesionales especializados en los campos contable-financieroeconómico, de ciencias ambientales de biología, de ingenieria, de derecho, de ciencias sociales, y de experto generalista de la industria o del gobierno)

\footnotetext{
9 MIGUEL CASAL, Armando y otros. Contabilidady Auditoria Medioambiental.p. 186.

10 Idem. p. 187.

11 MALHEIROS TELMA, Maria Marques, Brasil.

12 VALLECRIÓ, Eyer, Brasil.
} 
todos ellos con conocimiento de las normas y capacitación en la aplicación de los respectivos procedimientos de auditoría financiera $y$ de gestionn ${ }^{13}$.

Según Dense Pinheiro, la Auditoría Medioambiental «Es una evaluación sistemática para determinar si el sistema de control ambiental y el desempeño ambiental están de acuerdo con los programas de acción y si el sistema está siendo efectivamente implantado $y$ es adecuado para el cumplimiento de la politica ambiental de la empresais ${ }^{14}$.

Por otra parte, Rob Garay indica que la auditoría ambiental es: "una evaluación sistemática, objetiva y documentada del impacto de sus actividades del negocio sobre el ambienter) ${ }^{15}$.

\section{Naturaleza del Medio Ambiente}

Según Gonzáles Malaxechevarria ${ }^{16}$, la Auditoría Medioambiental consiste en la revisión exhaustiva de la instalación, procesos, almacenamientos, transporte, seguridad y riesgo, entre otros aspectos, que permitan definir planes de acción mediante los cuales se establezcan, con plazos determinados, las obras, reparaciones, correcciones, adquisiciones y acciones que puedan estar o no normados, pero cuya finalidad es la protección del medio ambiente.

En el ámbito del Mercado Único Europeo, a partir del $1^{\circ}$ de enero de 1993 , se vieron favorecidas aquellas industrias que adoptaron en sus procesos productivos medidas de protección al ambiente.
Como una consecuencia de esta política de fabricación-comercialización han surgido los eco productos; es decir,productos que desde su proceso de producción hasta su eliminación final, no son «agresivos» al ambiente. Es decir, la protección del ambiente ha sido rápidamente incorporada por el empresariado europeocomo un nuevo eimportantísimo factor de marketing comercial.

Este nuevo enfoque dado a la política ambiental se ha convertido en una política de tipo integradora de las demás políticas sectoriales, es decir, la económica, industrial, agrícola y de servicios. La protección del ambiente forma parte de la economía del mercado como un elemento más, convirtiéndose en un bien añadido a considerar. Este cambio de enfoque estratégico de los mercados, principalmente europeos y norteamericanos, posibilita la adecuación de los mismos al denominado «desarrollo sostenido», es decir, hacer compatible la utilización de los recursos naturales, el progreso, el desarrollo económico y la protección del ambiente.

En el ámbito europeo, las empresas que lideran en cuanto a la inversión destinada a la protección del ambiente, son las pertenecientes al sector químico, con aproximadamente un $40 \%$ del total de la inversión. Del total de esta inversión:

- El 50-60\% correspondería a la depuración del agua;

- El 25-30\%, al tratamiento de los residuos; y

3ONZÁLEZ MALAXECHEVARRIA, Ángel. Auditoria Ambiental: Su evolución bistórica y su entorno politicoinstitucional. España, 1998, p. 108.

- PINHEIRO FRANCISCO,Dense. Auditoría de control ambiental. Brasil.

GARAY, Rob y otros. Op.cit., p. 111.

GONZÁLEZMALAXECHEVARRIA, Ángel. Op. cit., p. 108. 
- El 10-15\% a la depuración de las emisiones a la atmósfera.

Estas nuevas tecnologías tienden a la introducción de cambios en los procesos de la fabricación con vistas a la reducción del consumo de materias primas generadoras de grandes cantidades de residuos y consumo de agua, el ahorro energético y al reciclado de agua y residuos, con el objeto de reducir la contaminación y los costes derivados a su corrección.

Todo esto es lo que ocurre en el ámbito europeo, en cambio en América Latina, aun no se ha llegado a este nivel y prácticamente estamos en una etapa de concientización y emisión de normatividad sobre el medio ambiente.

\section{Objetivos de la Auditoria del Medio Ambiente}

Los principales objetivos de la auditoría del medio ambiente son las que se indican a continuación:

- Proteger el ambiente y fomentar el desarrollo de una cultura ambiental empresarial.

- Establecer mecanismos para el cumplimiento de las normas legales.

- Investigar si las prácticas de gestión utilizadas son las aceptadas.

- Brindar un servicio técnico de orientación para la empresa.

- Motivar en los consumidores el hábito de adquirir productos fabricados por Industrias que observen prácticas de cuidado ambiental.

- Distinguir a las empresas que a través de la Auditoría y con el respectivo plan de acción, han aceptado la responsabilidad voluntaria de proteger a sus trabajadores, la comunidad y el ambiente mediante $e$ otorgamiento del certificado com: Industria Limpia.

Desde un punto de vista técnico, la

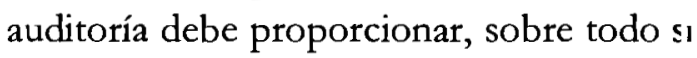
la situación así lo requiere, por su grado de complejidad, la mejor tecnología disponible. Esto implica la disposición por parte de. equipo auditor de los medios necesarios para poder ofrecer esta credibilidad y fiabilidad. lo que se traduce que necesita especialistas destacados en cada materia.

En la etapa de fijación de objetivos es importante lograr un acuerdo económico, ajustando el proyecto a las necesidades de la empresa. Se pueden presentar, tal como ocurre en el resto de las auditorías, proyectos alternativos a diferentes costes, de manera que se cumplan los objetivos legales, pero se puedan mejorar tecnológicamente con mayores inversiones en el futuro.

Posteriormente se pueden presentar objetivos específicos a cubrir en las diferentes áreas o departamentos, como puede ser el desarrollo de planes de integración del personal dentro de los objetivos generales, de modo que se comprenda la importancia del tema en cuestión y la necesidad de cumplir con los objetivos fijados. En este sentido, la formación continua debe considerarse prioritaria, por lo que el reciclaje a través de cursos, seminarios, conferencias., resulta vital para poder lograr un desempeño efectivo.

En otro orden de cosas se debe considerar la posibilidad de contar con la colaboración de otras empresas, para lo cual se definirán cuales deberán ser y en que grado o medida se establecerá la cooperación. Además de efectuar los contactos pertinentes (p.e.: con gabinetes 
jurídicos, asesores económico financieros, etc.), se debe contar con el apoyo de un sistema de gestión integrado y un programa comercial adecuado que facilite la actividad auditora.

\section{Importancia}

Para comprender la importancia de la auditoría medio ambiental debemos plantearnos las siguientes interrogantes: ¿Para qué debe emplearse la auditoría ambiental como una herramienta de gestión y evaluación? ¿Qué puede hacer una auditoría del medio ambiente por usted?

Porque la auditoría del medio ambiente contribuye a una adecuada gestión ambiental y cumplimiento de los deberes de la organización en cuanto a la legislación, reducción de costes y atender las presiones de los usuarios, ser más competitivos, mejorar su imagen corporativa en relación con su entorno.

Puede decirse que la razón básica para realizar una auditoría medioambiental es la supervivencia del ser humano, fundamentalmente para prolongar la estadía del hombre sobre la Tierra, por ello todas las leyes de los países se preocupan por la preservación, conservación y protección del medio ambiente. Si bien es cierto, cualquier otro tipo de auditoría es necesaria para conocer el estado de la empresa, la auditoría medioambiental es imprescindible, tambiẻn, por otros motivos: Cumplir la legislación, apaciguar la presión pública y evitar sanciones.

\section{Características}

Las características de la auditoría medio ambiental se sustentan en los procedimientos aplicados al examen y evaluación de los proyectos, programas actividades y operaciones que desarrollan las entidades sujetas al control del ente estatal, y los particulares para la gestión de las políticas ambientales de trascendencia nacional.

A partir de la Constitución Política del Estado, articulo $2^{\circ}$ que señala: «Toda persona tiene derecho a la paz, a la tranquilidad, al disfrute del tiempo libre y al descanso, asícomo a gozar de un ambiente equilibrado y adecuado al desarrollo de su vida»; y los artículos $60^{\circ}, 66^{\circ}$ y $89^{\circ}$, que regulan otros aspectos medio ambientales, la auditoría medio ambiental ha pasado a ser sujeto de evaluación en lo económico, financiero, contable, administrativo y jurídico, dando lugar a nuevos elementos de intervención, tales como el control de gestión, el control de resultados y el control físico de los bienes colectivos.

El control estatal (o fiscal) ambiental incluye la evaluación de la gestión de protección, uso, explotación, conservación de los recursos naturales y el medio ambiente, con fundamento en los principios de eficiencia, eficacia, equidad y economía

La aplicación del principio de eficiencia tiene por objeto establecer si, en igualdad de metas de cantidad y calidad, el gasto ambiental ejecutado por las empresas y entidades se realiza al mismo costo. Dicho de otro modo, determinar si la opción elegida entre alternativas equivalentes, para mitigar los costos ambientales de los proyectos de inversión, es la más económica.

El principio de eficacia determina si las metas y objetivos propuestos en los planes, políticas y programas de la empresas y entidades, en cuanto a recuperación, conservación, protección, ordenamiento, manejo, uso y aprovechamiento de los recursos naturales renovables y el medio 
ambiente, se cumplieron en términos de cantidad, calidad y oportunidad, y contribuyeron a garantizar el desarrollo sostenible.

Por su parte, el principio de equidad permite identificar los receptores de la acción económica ambiental y la distribución de gastos y beneficios entre sectores económicos y sociales, y entre entidades territoriales.

Finalmente, el principio de economía tiene por objeto determinar si la asignación de recursos para apoyar la gestión ambiental es la más conveniente para maximizar sus resultados.

\section{Ventajas de la Auditoría del Medio} Ambiente

La Auditoría Medioambiental lleva consigo una serie de ventajas para la empresa o para la sociedad auditada, como pueden ser:

a) La mejora del rendimiento y la utilización de los recursos, con lo cual se produce un incremento en el ahorro.

b) La posibilidad de utilizar una valiosa información ambiental en la toma de decisiones continua que permita, ante cualquier cambio, efectuar las mediciones de impacto ambiental, que provocaría la nueva estrategia a poner en práctica. Del mismo modo, ante cualquier situación de emergencia que pudiera producirse, supone contar con un apoyo informativo seguro y eficaz que permite dirigir los esfuerzos en la dirección acertada.

c) La facilidad que proporciona para obtener seguros que cubren riesgos ambientales, así como para la obtención de licencias, permisos, ayudas c subvenciones, contratos públicos, etc.

d) Ayuda tanto a directivos como a empleados en el conocimiento de la situación y política medioambiental $\mathrm{dc}$ la empresa, facilitando además el intercambio de información entre lcs diversos sectores productivos. Esto es posible gracias a la elaboración de un informe final, documento que se entrega a la dirección y que ésta utilizará comc herramienta de control interno. Esta información, además, podrá ser manejada por todo el personal de la empresa, los organismos oficiales correspondientes y, en general, por cualquier interesado en conocer la situación medioambiental de la empresa.

e) Permite mejorar la salud, seguridad e higiene de los trabajadores.

\section{Participantes en el proceso de auditoría de medio ambiente}

Participan los siguientes agentes:

- Empresa.

- Sociedad o firma auditora contratada por la Empresa.

- Prestadores de servicios. (Laboratorio: de Pruebas a residuos, análisis de aguas residuales, emisiones a la atmósfera. remediación de suelos etc.).

- Entidades rectoras del medio ambiente. Institución supervisora o controladora creada especialmente -sociedad mixtaen México como la PROFEPA.

\section{Estructura de la Auditoría Ambiental}

La auditoría medioambiental comprende las etapas y actividades siguientes: 
a) Pre auditoría - Etapa de planeación

- Asignación y contratación del Auditor y Supervisor.

- Establecimiento de un Convenio de Confidencialidad entre Empresa, Auditor y Supervisor.

- Auditor prepara una Propuesta Técnico-Económica que debe ser revisada por la Empresa, y aprobada porla entidad o sector rector del medio ambiente.

- Recopilación de información en planta.

- El auditor prepara un Plan de Auditoría que debe ser revisado por la Empresa y aprobado por el sector de la empresa al que pertenece.

b) Auditoría - Etapa de ejecución

- Auditoría en campo (instalaciones y documentación).

\section{c) Postauditoría- Etapa de reporte y seguimiento}

- El auditor prepara un Reporte de Auditoría que debe ser revisado por la Empresa y aprobado por el sector correspondiente al que pertenece la empresa.

- La empresa prepara un Plan de Acción que debe ser aprobado por la entidad rectora del medioambiente.

- La empresa y la entidad rectora suscriben el convenio de cumplimiento.

- La empresa entrega a la entidad rectora del medio ambiente los reportes de avance trimestrales hasta completar todos los puntos del Plan de Acción.
- Cierre del Proceso de Auditoría Ambiental.

10. ¿En qué participamos los Contadores Públicos?

Con excepción del Ingeniero Ambiental, que participa durante todo el proceso de Auditoría, los demás departamentos participan principalmente en las siguientes actividades:

- Recopilación de información (Etapa de Planeación).

- Auditoría en campo (Etapa de Ejecución).

a) Recopilación de Información

- Definir estándares y procedimientos (éstos pueden ser gubernamentales o establecidos por nosotros mismos).

- Debe existir documentación que acredite el cumplimiento de los estándares y procedimientos aceptados.

- En general, el carácter de la información requerida guarda mucha similitud con ISO-9000 ( «da lo que haces, haz lo que dices y compruébalo»), ya que el proceso de Auditoría ambiental está fuertemente influenciado por la Norma ISO-1400014001.

b) Auditoría en Campo

- Tiene por objeto verificar el cumplimiento de los procedimientos $y$ estándares previamente definidos.

- Únicamente los responsables están autorizados a proporcionar la información requerida por los auditores.

- Hablar con la verdad, procurando contestar únicamente lo que se pregunta.

- Participar en las Juntas de Inicio y Cierre de Actividades. 
11. Normas de la Auditoría del Medio Ambiente

\section{a) Normas UNE}

Es una especificación técnica de aplicación voluntaria aprobada por AENOR, organismo de Normalización, certificación reconocida. (R.D. 1614/ 1985; R.D. 2200/1995) en desarrollo de la ley 21/1992 de Industria.

Con la aparición de las normas UNE que indican las reglas generales para el desarrollo de un Sistema de Gestión Medioambiental y el desarrollo de las Normas ISO 14000 ha provocado un aumento por parte de las empresas del interés hacía la auditoría medioambiental. Todo ello hace pensar en un rápido e importante desarrollo de las auditorías medioambientales en este sentido.

\section{b) Normas ISO 14000}

Según Quijano ${ }^{17}$, la ISO 14000 es el nombre genérico del conjunto de normas ambientales creadas por la TC 207 de la ISO (Intenational Organization for Standarization). ISO 14000 es una serie de standards internacionales, que especifica los requerimientos para preparar y valorar un sistema de gestión que asegure que su empresa mantiene la protección ambiental y la prevención de la contaminación en equilibrio con las necesidades socio-económicas.

Dentro de las diversas normas publicadas, la ISO 14000, norma de Sistemas de Gestión Ambiental, es la más conocida y la única que se puede certificar. De esta forma, la certificación del suplemento 14001 es la evidencia que las Empresas poseen un Sistema de Gestión Ambiental (SGA) implementado, pudiendo mostrar a través de ella su compromiso con el medio ambiente.

La ISO 14000 no es una ley en el sentido que nadie se exige ser registrado; sin embargo, nadie obliga a nadie a comprar sus productos y servicios, pero se debe estar preparado si en el otro país se ha declarado ISO 14000 como requisito para hacer negocio. Ésta es una barrera de comercio legal reconocida bajo el tratado internacional.

ISO 14000 realmente es una serie de normas que cubren todos los sistemas de dirección medioambiental (El SME). Cascio $^{18}$ dice que la ISO 14001 requiere conformidad con una serie de elementos de un SME. Es decir, la organización debe mostrar que tiene un sistema del funcionamiento en lugar de producir los resultados requeridos. El ISO 14001 no dicta cómo se hace esto, pero exige a una auditoría severa determinar que ellos se hacen de hecho y están operando continuamente. ISO 14001, por ejemplo, no requiere que una organización esté conforme a cualquier ley medioambiental, pero requiere que la organización sepa a qué regulaciones está sujeta, y tiene en lugar un sistema comprobable por lograr complacencia y por encabezar fuera de los incumplimientos antes de que ellos ocurran.

17 QUIJANO PONCEDELEÓN, Andrés. Normas ISO 14000. Bogotá, Colombia.

${ }^{18}$ CASCIO,Joseph; WOODSIDE, Gayle y Mitchell PHILIP. Guia ISO 14000: las nuevas normas internacionalespara la administración ambiental. México, McGraw-Hill, 1997. 
Esto plantea otro aspecto de ISO 14001: los aspectos medioambientales. Este elemento del comandante de ISO 14001 requiere que una organización sepa qué impactos está teniendo en el ambiente. Este conocimiento debe ir más allá del conocimiento del libro de texto, no más de mando de polución típico. Debe tener en cuenta los aspectos medioambientales de la facilidad específica peculiar a sus funcionamientos, procesos, productos, ysu situación. El objetivo es identificar los «aspectos» medioambientales $y$ continuamente trabajar para minimizar efectos negativos de funcionamiento. Ésta es la llave a ISO 14001: un sistema de dirección que asegura a la organización entera que está envuelta en una mejora incesante. El sistema debe tener una estructura que fuerza, mejora, y puede demostrarlo.

En la actualidad, a nivel mundial, las normas ISO 9000 e ISO 14000 son requeridas, debido a que garantizan la calidad de un producto mediante la implementación de controles ex-haustivos, asegurándose deque todos los procesos que han intervenido en su fabricación operan dentro de las características previstas.

Toda empresa debe tener en cuenta estas normas, pues son el punto de partida en la estrategia de la calidad, así como para la posterior certificación de la empresa.

Todas las normas de la familia ISO 14000 fueron desarrolladas sobre la base de los siguientes principios:

c) Deben resultar en una mejor gestión ambiental;

d) Deben ser aplicables a todas las naciones;

e) Deben promover un amplio interés en el publico y en los usuarios de los estándares; f) Deben ser costo efectivas, no prescriptivas y flexibles, para poder cubrir diferentes necesidades de organizaciones de cualquier tamaño en cualquier parte del mundo;

g) Como parte de su flexibilidad, deben servir a los fines de la verificación tanto interna como externa;

h) Deben estar basadas en conocimiento científicos; y por sobre todo, deben ser prácticas, útiles y utilizables.

12. Organismos responsables del Medio Ambiente en el Perú

\section{a) El Instituto Nacional de Recursos Naturales - INRENA}

El Instituto Nacional de Recursos Naturales-INRENA es un organismo público descentralizado del Ministerio de Agricultura, creado por Decreto Ley $\mathrm{N}^{\circ}$ 25902, el 27 de noviembre de 1992, encargado de realizar las acciones necesarias para el aprovechamiento sostenible de los recursos naturales renovables, cautelar la conservación de la gestión sostenible del medio ambiente rural y la biodiversidad silvestre. Como autoridad nacional, debe realizar su trabajo en estrecha relación con gobiernos regionales y locales, sociedad civil organizada e instituciones públicas y privadas. Brinda servicios de concesiones forestales con fines maderables y no maderables (otros productos del bosque, ecoturismo y conservación), autorizaciones y permisos forestales, control forestal, autorizaciones de ingreso a las áreas naturales protegidas con fines de investigación científica, filmica o fotográfica, información sobre la gestión de recursos hídricos, autorizaciones para el funcionamiento de zoocriaderos y áreas de manejo de fauna silvestre, permisos de exportación 
de flora y fauna silvestre, evaluación ambiental, entre otros.

El Perú es uno de los diecisiete países con mayor diversidad biológica en el mundo; correspondiéndole desempeñar un papel preponderante en la conservación de sus recursos naturales. De allí que el Sistema Nacional de Áreas Naturales Protegidas por el Estado (SINANPE), tiene especial trascendencia.

Las áreas naturales protegidas son espacios continentales y/o marinos del territorio nacional reconocidos, establecidos y protegidos legalmente por el Estado como tales, debido a su importancia para la conservación de la diversidad biológica y demás valores asociados de interés cultural, paisajístico y científico, así como por su contribución al desarrollo sostenible del país ${ }^{19}$.

Los ecosistemas, también, son tan frágiles, según expertos del INRENA, bajo una seria amenaza por causa del desarrollo humano, la colonización de tierras y la depredación de recursos naturales, revela que hasta el momento se ha logrado proteger más del 13\% de la superficie del país. Sin embargo, en 12 áreas protegidas que son consideradas prioritarias, no se realizan ningún tipo de cobertura (léase estudios, investigaciones, registros $\mathrm{y}$, por cierto, labores de protección) por falta de recursos. Similar realidad se presenta en los ecosistemas marinos de islas y puntas. Como área protegida en el litoral de la Costa, solo existe una reserva nacional que es Paracas y no hay, como en otros países, ningún área natural protegida submarina. Gran parte de los pastizales y bofedales altoandinos se encuentran también en una situación de gran precariedad ${ }^{20}$.

\section{b) EI Consejo Nacional del Medio} Ambiente - CONAM

Es la autoridad ambiental nacional que tiene por finalidad planificar, promover, coordinar, controlar y velar por el ambiente y el patrimonio natural de la Nación. El CONAM fue creado mediante Ley $\mathrm{N}^{\circ}$ 26410, publicada el 22 de diciembre de 1994. Tiene como objetivo promover la conservación del ambiente, a fin de coadyuvar al desarrollo integral de la persona humana sobre la base de garantizar una adecuada calidad de vida, propiciando el equilibrio entre el desarrollo socioeconómico, el uso sostenible de los recursos naturales y la conservación del ambiente. Constituye un organismo público descentralizado adscrito al ámbito de la Presidencia del Consejo de Ministros ${ }^{21}$.

\section{RESULTADOS MÁS SALTANTES}

Los resultados de la presente investigación sobre Auditoría Del Medio AMBIENTE es de aplicación por los contadores públicos, así como de otros profesionales dedicados a la gestión y preservación del medio ambiente: administradores, economistas, biólogos y otros especialistas; así como de sociedades, asesores y consultores de empresas dedicadas a

tut cio ursos Naturales. [Enlínea]. Disponible en: http://www.inrena.gob.pe/index_inicio.htm NECOCHEA FLORES, Carlos. «Ecosistemas peruanos se encuentran amenazados por el avance del hombre» En:ElComerczo. 20-05-2006,pp. B12.

EI Consejo Nacional del Medio Ambiente - CONAM [En línea]. Disponible en: http://www.conam.gob.pe/ modulos/home/queeselconam.asp 
actividades de Auditoría de Medio Ambiente. De la misma manera, será de aplicación para las universidades, organismos de supervisión y control, como: la Contraloría General de la República, el Ministerio de Agricultura: Instituto Nacional de Recursos Naturales - INRENA, Consejo Nacional del Ambiente - CONAM.

También, los resultados de la investigación sobre Auditorúa DEL Medio Ambiente serán de aplicación por las organizaciones o empresas en general, involucradas en la explotación de los recursos naturales, prevención y conservación del medio ambiente.

Asimismo, los resultados de la presente investigación tendrán los aportes siguientes para los usuarios y la sociedad en general:

- Contribuirá a incrementar la calidad de gestión y preservación del medio ambiente.

- Proteger y prevenir la contaminación del medio ambiente para mantener el estándar de calidad de vida de todos los seres vivientes, y de esta manera prolongar la corta estadía del hombre en el planeta Tierra.

- Reducir y prevenir los altos costos sociales y económicos, generados por la contaminación ambiental o la degradación de nuestro planeta.

- Contribuirá a crear conciencia en la ciudadanía y en las empresas dedicadas a la explotación de los recursos naturales, y en todas aquellas empresas industriales que esta actividad se lleve a cabo de forma mesurada y racional dentro de la actividad económica.

\section{IMPACTO O APORTE DEL ESTUDIO}

$\mathrm{El}$ aporte del presente estudio puede ser utilizado por contadores y auditores, especialmente de empresas productoras y generadoras de contaminantes, ya que la auditoría medioambiental comprende la evaluación sistemática, documentada, periódica y objetiva del funcionamiento del sistema degestión destinados a la protección del medio ambiente y del cumplimiento de las disposiciones reglamentarias en vigencia.

El presente estudio tiene un aporte científico, social hacia la concepción de una metodología, marco teórico en cuanto a la auditoría del medio ambiente en la formación y educación continua de los auditores y consultores de empresas.

El presente estudio tiene un impacto técnico-profesional, social, institucional y empresarial.

Los beneficios y bondades del presente estudio serán utilizados por los contadores públicos y profesionales, en general, dedicados a la actividad de auditoría del medio ambiente, las universidades, colegios profesionales, docentes universitarios, estudiantes, empresas e instituciones, asesores y consultores de empresas y auditores.

También será de utilidad para entidades públicas y privadas, dedicadas a la explotación y conservación de los recursos naturales y la ecología en general.

La evaluación medio ambiental se considera como un estudio exhaustivo de los problemas generales de las actividades, servicios y proyectos, y su impacto en el medio 
ambiente, y de las medidas empleadas hasta ahora para controlarlas. Por ello, los esfuerzos de investigación en este tema novedoso constituyen un asidero y estímulo social.

\section{CONCLUSIONES Y RECOMEN- DACIONES}

Las conclusiones y recomendaciones de la presente investigación podrán ser utilizadas por los contadores y auditores, quienes deben capacitarse constantemente en Auditoría Medioambiental, con el objetivo de medir el grado de eficacia de la gestión medioambiental que aplican. Las cuales se mencionan a continuación

1. El auditor medioambiental identificará desde el inicio de la Auditoría las actividades y procesos que generan contaminantes. Se documentará y analizará en detalle el ciclo completo de cada uno de ellos, para efectos del planeamiento de la acción de control.

2. Los Tratados y Convenios Internacionales de preservación del medioambiente deben ser divulgados a todo nivel, estableciendo mecanismos de gestión y seguimiento para su cumplimiento, porque el éxito de la implementación de un sistema de gestión medioambiental depende primordialmente de que dicho sistema concuerde con lo estipulado en estos convenios y tratados establecidos en Normas de Gestión Medioambiental.

3. Incursionando en la auditoría medioambiental se contribuirá al desarrollo económico y social del país en problemas de gran relevancia como el de la contaminación medioambiental, si se tiene en cuenta que la gestión empresarial en este ámbito se encuentra en una etapa de desarrollo inicial y requiere el apoyo de los auditores para el cumplimiento de la legislación ambiental y lograr elevar la calidad de vida.

4. La auditoría del medio ambiente está dirigida a la evaluación de la gestión del medio ambiental de las empresas o instituciones, implicadas en el daño del medio ambiente, por los distintos procesos $y / 0$ actividades que realizan.

5. La preservación y conservación del medio ambiente está relacionada con la cultura y la filosofía a cerca de la vida del hombre en la tierra, ya que hoy en día los empresarios $y / o$ ciudadanos no valoramos la trascendencia del daño que produce a la ecología.

6. Los distintos tópicos de la auditoría aplicada al medio ambiente se hallan institucionalizados y organizados técnica y científicamente, como una herramienta moderna para la gestión empresarial.

7. En cuanto a la actividad académica, servirá de base a las universidades y colegios profesionales, para incluirse en sus currículas de estudio.

\section{REFERENCIAS}

American Accountant Association (citado por Walter Kell y William Boynton «Auditoría Moderna». Editorial CECSA, México 1999).

CASAL, Armando Miguel y otros. Contabilidad y Auditoria Ambiental. Ediciones Macchi, Buenos AiresArgentina, 2000. 
CASCIO, Joseph; WOODSIDE, Gayle y Mitchell PHILIP. Guia ISO 14000: las nuevas normas internacionales para la administración ambiental. México, McGraw-Hill, 1997.

CLEMENTS, Richard B. Guia completa de las normas ISO 14000. Barcelona, Gestión 2000, 1997.

CONESA FERNÁNDEZ, Vicente. Auditorias Medioambientales. Guias Metodológicas. Ediciones Mundi-Prensa, 1997.

Contraloría General de la República. «Manual de Auditor Gubernamental», Lima -Perú, 1998.

Federación de Colegios de Contadores Públicos del Perú -Junta de Decanos 2000. «Normas Internacionales de Auditoría -NIAS», 3. ${ }^{\text {ra }}$ ed., Lima - Perú.

FOY VALENCIA, P. Derecho Ambiental. Fondo Editorial de la Pontificia Universidad Católica del Perú, 1997.

WOODSIDE, Gayle y AURRICHIO, Patrick. Auditoria de Sistemas de Gestión Medioambiental-Introducción a la norma ISO 14001. McGraw-Hill / Interamericana de España, S. A. U., 2001.

GONZÁLEZ MALAXECHEVARRIA, Angel. Auditoria Ambiental: Su evolución bistórica y su entorno político-institucional. España 1998, p.108.

GRAY, Rob; BEBBINGTON, Jan y WALTERS, Diana. Contabilidad $y$ Auditoría Ambiental. Bogotá, ECOE Ediciones, 1999.

HERNÁNDEZ SAMPIRI, Roberto; FERNÁNDEZ COLLADO, Carlos y BAPTISTA, Pilar. Metodología de la Investigación. McGraw-Hill Interamericana México S.A., 1997.

ISO 14043 interpretación de la evaluación del. ciclo de vida. Ediciones Díaz de Santos S.A., Madrid-España, 1992.
Ley Orgánica del Sistema Nacional de Control y de la Contraloría General de la República. Ley No 27785.

MANTILLA PINILLA, Eduardo. "La contabilidad ambiental en el desarrollo sostenible». En: Revista Internacional Legis de Contabilidad \& Auditoria. Bogotá, enero-marzo 2006, pp.133-159.

MILLS, David. Manual de la Auditoría de la Calidad. Ediciones Gestión 2000 S.A., Barcelona, 1997.

NECOCHEA FLORES, Carlos. «Ecosistemas peruanos se encuentran amenazados por el avance del hombre». En: El Comercio. 20-05-2006, pp. B12.

OROPEZA MONTERRUBIO, Rafael. «Manualpráctico de Auditorías Ambientales». México 1997. p. 164.

PINHEIRO FRANCISCO, Dense. Auditoría de Control Ambiental. Brasil.

POLAR FALCÓN, E. 2000. «Auditoría Medio Ambiental: una Especialidad Necesaria de Conocer y Aplicar en el Perú). En: Revista $N^{\circ} 13$ Quipukamayoc del Instituto de Investigación de Ciencias Financiera y Contables de UNMSM.

Procuraduría Federal de Protección del Medio Ambiente - PROFA, 1992, México.

QUIJANO PONCE DE LEÓN, Andrés. Normas ISO 14000. Bogotá, Colombia.

GRAY, Rob y otros. Contabilidady Auditoría Ambiental. Litoperla Impresiones Ltda., Colombia, 2001.

TORRES BARDALES, C. El proyecto de investigación científica. Editorial San Marcos, Lima -Perú, 1998.

Trabajos Técnicos Nacionales e Internacionales, Conferencia Interamericana de Contabilidad, XXIV Conferencias, Punta del Este, Uruguay, 2001. 
Trabajos Técnicos Nacionales e Internacionales, Conferencia Interamericana de Contabilidad, XXIII Conferencias, San Juan de Puerto Rico, 1999.

Unión Europea - UE. Eco. Management and audit. Scheme (EM-AS).

Valle Crió Eyer. Brasil.

WAINTEIN, Mario y CASAL, Armando Miguel: «La auditoría Integral en el marco de un mundo globalizado», XXIII
Conferencia Interamericana de Contabilidad, San Juan de Puerto Rico, del 02 al 05 de Agosto de 1999.

WAINTEIN, Mario y CASAL, Armando Miguel: Normas de Auditoria Nacionales Internacionales. Desarrollo y Gestion. Profesional y Empresarial. Ediciones Errepar, Ciudad Autónoma de Buenos Aires, septiembre del 2000. 